\title{
Sustained oscillations in stochastic systems
}

\author{
Juan Pablo Aparicio *, Hernán Gustavo Solari ${ }^{1}$ \\ Departamento de Física, Facultad de Ciencias Exactas y Naturales, Universidad de Buenos Aires, 1428 Buenos Aires, \\ Argentina
}

Received 29 February 2000; received in revised form 8 September 2000; accepted 10 October 2000

\begin{abstract}
Many non-linear deterministic models for interacting populations present damped oscillations towards the corresponding equilibrium values. However, simulations produced with related stochastic models usually present sustained oscillations which preserve the natural frequency of the damped oscillations of the deterministic model but showing non-vanishing amplitudes. The relation between the amplitude of the stochastic oscillations and the values of the equilibrium populations is not intuitive in general but scales with the square root of the populations when the ratio between different populations is kept fixed. In this work, we explain such phenomena for the case of a general epidemic model. We estimate the stochastic fluctuations of the populations around the equilibrium point in the epidemiological model showing their (approximated) relation with the mean values. (c) 2001 Elsevier Science Inc. All rights reserved.
\end{abstract}

Keywords: Non-linear dynamics; Stochastic oscillations; Population dynamics; Interacting populations

\section{Introduction}

Interacting populations are common in ecology and epidemiology as well as in many other areas of natural sciences. Usually, the dynamics are described by a system of coupled deterministic differential equations whose solutions may present damped oscillations. However, stochastic simulations show that the oscillations may persist, with amplitudes considerably larger than the square root of the mean value [1]. The dynamics of interacting populations are, in general, a stochastic process. In view of this we must ask: What is the validity of the deterministic de-

\footnotetext{
${ }^{*}$ Corresponding author. Present address: Department of Biometrics, 432 Warren Hall, Cornell University, Ithaca, NY 14853-7801, USA. Tel.: +1-607 255 8103; fax: +1-607 2554698.

E-mail addresses: jpa9@cornell.edu (J.P. Aparicio), solari@df.uba.ar (H.G. Solari).

${ }^{1}$ Fax: +54-11 45763357.
} 
scription? Why do oscillations not die out? How do the mean oscillation amplitudes depend on the parameters?

In this work, we answer these questions for a simple but frequently found two-dimensional system. We consider two interacting populations of time-dependent size $N(t)$ and $n(t)$. We assume that the dynamics are well captured by the following model:

- The $N$-population receives a constant flux $\Omega a$ ('birth').

- The populations interact at rate $\beta N n / \Omega$, with $\beta$ constant. After each encounter, the $N$-population decreases by one while the $n$-population increases by one ('contagion').

- The per capita removal rate in the $n$-population is $b$ ('death').

This system has been widely used in epidemic models (see [2] for a critical review), and is similar to those used for modeling predator-prey interactions [1], chemical kinetic reactions [3] or laser light-carrier interactions [4]. The deterministic version was used by Soper [5] to study the periodicity of measles outbreaks. The stochastic counterpart was developed by Bartlett [6,7]. Inclusion of seasonality [8] or a latency period not exponentially distributed [9] allows a better match with field data. In some of these works, 'initial' conditions are such that extinction after an outbreak is almost certain, and recurrence is due to an infected population flux (see also [1, p. 341]); in this context, the expected time to extinction plays a central role.

In spite of the simplicity of the system, there is no exact solution for it. Approximate results were obtained by means of the diffusion approximation $[2,10]$.

In this work, we study the behavior of the solutions of the stochastic version of the model when extinction is unlikely. We find that there is a region of the phase space, $(N, n)$, for which the deterministic description is absolutely inappropriate. Depending on the ratio of the mean values of the populations at equilibrium, this region may be significantly large, its size relative to the equilibrium populations diminishes with the 'size' of the system (see the parameter $\Omega$ below, Eqs. (1)). This result may have consequences in the design of experiments testing density-dependence regulation or in the analysis of temporal series.

The rest of the article is organized as follows: in Section 2, we introduce the deterministic model; in Section 3, we present and analyze the stochastic model; Section 4 presents a summary and discussion of results, while Section 5 presents the concluding remarks.

\section{Deterministic description}

In this section we briefly review the deterministic model, presenting the results that will be useful later. The model reads

$$
\begin{aligned}
& \frac{\mathrm{d} N}{\mathrm{~d} t}=a \Omega-\beta N n / \Omega, \\
& \frac{\mathrm{d} n}{\mathrm{~d} t}=\beta N n / \Omega-b n .
\end{aligned}
$$

The parameter $\Omega$ plays the role of a scale factor, i.e., the time evolution of the variables $N / \Omega$ and $n / \Omega$ does not depend on $\Omega$. The introduction of the scale parameter in (1) is particularly useful to relate the deterministic and the stochastic dynamics (see, for example, [11, Chapter IX]) and is usually associated with the total population size (see, for example, [2]). 
Model (1) has only one equilibrium given by $N_{\mathrm{eq}}=\Omega b / \beta, n_{\mathrm{eq}}=\Omega a / b$. Linearization of the system around the equilibrium values leads to

$$
\begin{aligned}
& \frac{\mathrm{d} x}{\mathrm{~d} t}=-\beta / \Omega\left(n_{\mathrm{eq}} x+N_{\mathrm{eq}} y\right)=-(\beta a / b) x-b y, \\
& \frac{\mathrm{d} y}{\mathrm{~d} t}=\beta / \Omega\left(n_{\mathrm{eq}} x+N_{\mathrm{eq}} y\right)-b y=(\beta a / b) x,
\end{aligned}
$$

where we have defined $x=N-N_{\text {eq }}$ and $y=n-n_{\text {eq }}$.

The linear set of equations (2) is equivalent to the second-order differential equation

$$
\frac{\mathrm{d}^{2} x}{\mathrm{~d} t^{2}}=-(\beta a / b) \frac{\mathrm{d} x}{\mathrm{~d} t}-\beta a x=-\gamma \frac{\mathrm{d} x}{\mathrm{~d} t}-\omega_{0}^{2} x
$$

for a damped oscillator of damping ratio $\gamma=\beta a / b$. Here, $\omega_{0}=\sqrt{\beta a}$ represents a characteristic frequency.

The existence of damped oscillatory solutions requires that $\omega_{0}>\gamma / 2$, which is equivalent to the condition

$$
\alpha^{2} \equiv \frac{b^{2}}{\beta a}=\left(\frac{\omega_{0}}{\gamma}\right)^{2}=\frac{N_{\mathrm{eq}}}{n_{\mathrm{eq}}}>\frac{1}{4} .
$$

The period of oscillations is

$$
\tau=\frac{2 \pi}{\omega_{0}} \frac{1}{\sqrt{1-\left(1 / 4 \alpha^{2}\right)}} .
$$

The stability of the fixed point in the linear approximation can be asserted using Liapunov's first stability criteria (see, for example, [12]) by considering the Liapunov function

$$
E=x^{2}+\alpha^{2} y^{2} .
$$

Note that constant values of $E$ determine (deformed) circles in the phase space $(N, n)$ of radius $r=$ $\sqrt{E}$ (the circles can be seen scaling $n$ by $\alpha$ ). The variable $r$ can also be considered as a coordinate of the system, and in such a case, the complementary coordinate is the angle $\theta=\arctan (\alpha y / x)$, which is an increasing function of time under Eq. (3).

The Liapunov function $E$ is non-negative and equals zero only at the equilibrium point $(x, y)=(0,0)$; moreover, its derivative along the linearized flow (3) is

$$
\frac{\mathrm{d} E}{\mathrm{~d} t}=-2 \beta a / b x^{2}=-2 \gamma x^{2} .
$$

As such, it suffices to prove the local stability of the equilibrium point under the linear approximation. The Liapunov function $E$ can then be extended to a Liapunov function of the non-linear flow by adding higher-order terms. However, standard considerations [13] allow us to build a oneparameter family of Liapunov functions, $E_{\Gamma}$, for $0<\Gamma<1$, of the form

$$
E_{\Gamma}=\left((1-\Gamma) x^{2}+\alpha^{2} y^{2}\right)+\Gamma(x+y)^{2},
$$


which is non-negative and takes the value zero only at the equilibrium point as required in Liapunov's theorem (the function $E$ is a limit point for this family of functions). The derivative of $E_{\Gamma}$ following the non-linear flow (1) reads

$$
\frac{\mathrm{d} E_{\Gamma}}{\mathrm{d} t}=-2 \gamma(1-\Gamma)\left(\frac{y}{n_{\mathrm{eq}}}+1\right) x^{2}-2 b y^{2}\left(\Gamma-\frac{x}{n_{\mathrm{eq}}}\right),
$$

which is negative defined in the rectangular region

$$
\left\{(x, y) \text { such that }-N_{\mathrm{eq}}<x<\Gamma n_{\mathrm{eq}},-n_{\mathrm{eq}}<y<n_{\mathrm{eq}}\right\}
$$

and takes the value zero only at the equilibrium point $(0,0)$. The function $E_{\Gamma}$ satisfies all the requirements for a Liapunov function to imply (local) asymptotic stability of the equilibrium point.

We will later study the evolution of the Liapunov functions $E$ under the stochastic dynamics and briefly comment on the differences that appear when $E_{\Gamma}$ is considered.

\section{Stochastic description}

The stochastic model is produced by considering three independent events: 'birth' in the $N$-population, 'contagion', and 'death' in the $n$-population. The effect of each event on the population numbers and the corresponding transition rate for the probabilities are summarized in Table 1.

The forward Kolmogorov (or master) equation for the probability distribution reads

$$
\frac{\mathrm{d} P_{N, n}}{\mathrm{~d} t}=W_{b}^{N-1, n} P_{N-1, n}+W_{c}^{N+1, n-1} P_{N+1, n-1}+W_{d}^{N, n+1} P_{N, n+1}-\left(W_{b}^{N, n}+W_{c}^{N, n}+W_{d}^{N, n}\right) P_{N, n} .
$$

We will understand this equation as being valid for all possible integers values of $n$ and $N$ and will restrict attention to the realistic cases where only a non-negative number of individuals can be found, i.e., we will only accept initial conditions with $P_{N, n}=0$ whenever $N<0$ or $n<0$. The Kolmogorov equation propagates this property to all times, since the death rate is zero when $n=$ 0 and the contagion rate is also zero when $n=0$ or $N=0$.

Individual realizations of the stochastic process are simulated with event probabilities $P_{b}=W_{b}^{N, n} / R, P_{c}=W_{c}^{N, n} / R$ and $P_{d}=W_{d}^{N, n} / R$, where $R=W_{b}^{N, n}+W_{c}^{N, n}+W_{d}^{N, n}$, while the inter-event times are given by a random variable $\Delta t$ exponentially distributed with mean $1 / R[1]$.

In Fig. 1, we show a stochastic simulation compared to the deterministic solution. It can be seen that, for a time of approximately $3 \tau$, both solutions are almost identical. Then, the deterministic

Table 1

\begin{tabular}{lll}
\hline Event & Effect & Transition rate \\
\hline Birth & $(N, n) \rightarrow(N+1, n)$ & $W_{b}^{N, n}=\Omega a$ \\
Contagion & $(N, n) \rightarrow(N-1, n+1)$ & $W_{c}^{N, n}=\beta N n / \Omega$ \\
Death & $(N, n) \rightarrow(N, n-1)$ & $W_{d}^{N, n}=b n$ \\
\hline
\end{tabular}




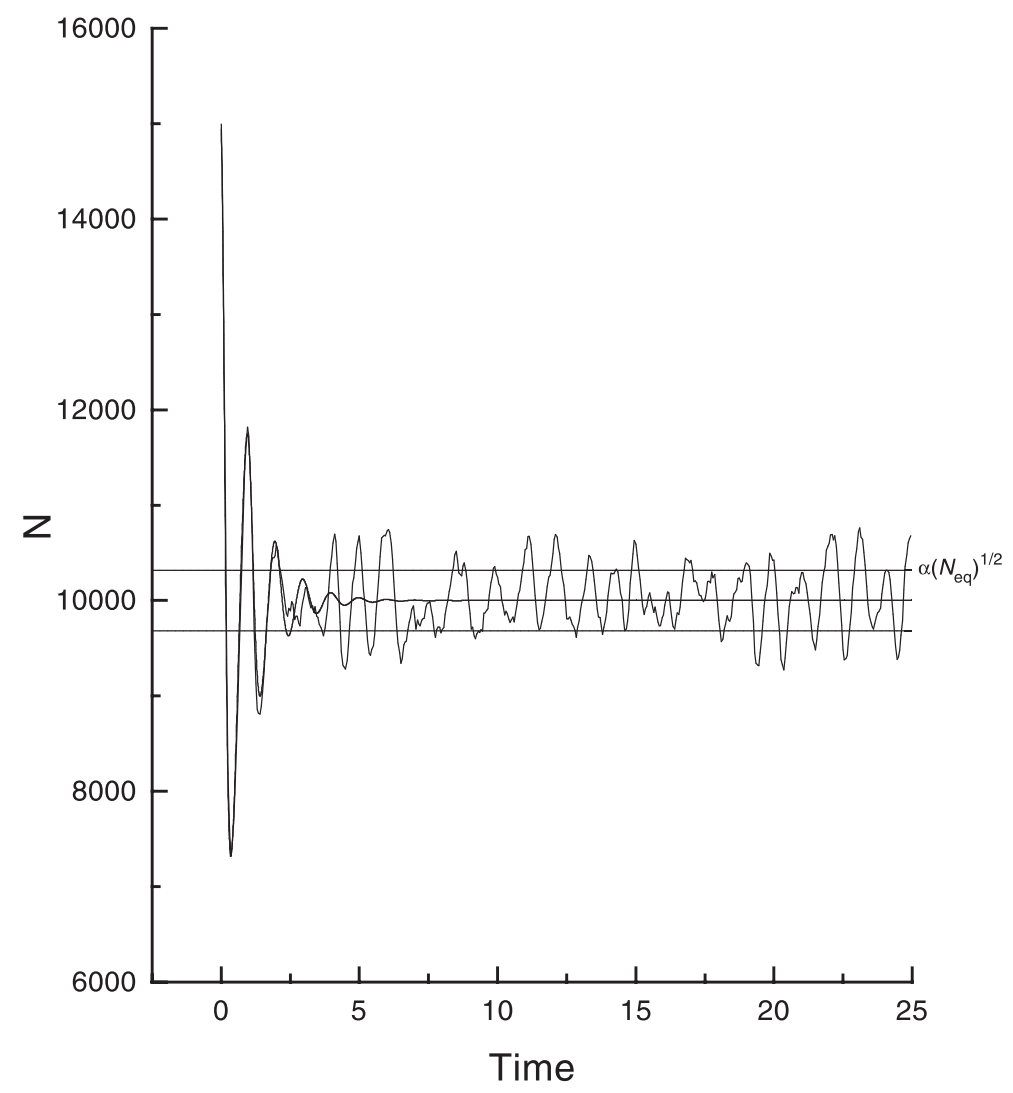

Fig. 1. Deterministic solution and stochastic simulation. The deterministic equilibrium values are $N_{\mathrm{eq}}=10^{4}, n_{\mathrm{eq}}=10^{3}$, hence $\alpha=\sqrt{10}$. The time unit is the period of the deterministic solutions $\tau \simeq \tau_{0}=2 \pi / \sqrt{\beta a}$. The horizontal lines are placed at $N=N_{\text {eq }} \pm \alpha \sqrt{N_{\text {eq }}}$. Parameter values are $a=1, b=10, \beta=10, \Omega=10^{4}$.

oscillation 'dies out', while the stochastic simulation presents 'undamped oscillations'. In Fig. 2, we start a stochastic simulation from the deterministic equilibrium values, and we can see that an oscillatory regime is soon established. A simple explanation of this behaviour is given in the following.

We will now focus our attention on the geometry of the trajectories, and hence, we map the stochastic process into an embedded stochastic process with constant time interval between events.

Close to the deterministic equilibrium values, the three events have almost the same probability of occurrence since

$$
a \Omega=\beta N_{\text {eq }} n_{\text {eq }} / \Omega=b n_{\text {eq }} .
$$

The population state close to the equilibrium point $\left(N_{\text {eq }}, n_{\text {eq }}\right)$ performs a random walk in phase space whose three possible steps are along the axes or the diagonal, the three events having almost the same probability of occurrence. As a consequence, we expect the distance from the population state to the deterministic equilibrium value to be of the order of $\sqrt{M}$ after $M$ steps. This behavior 


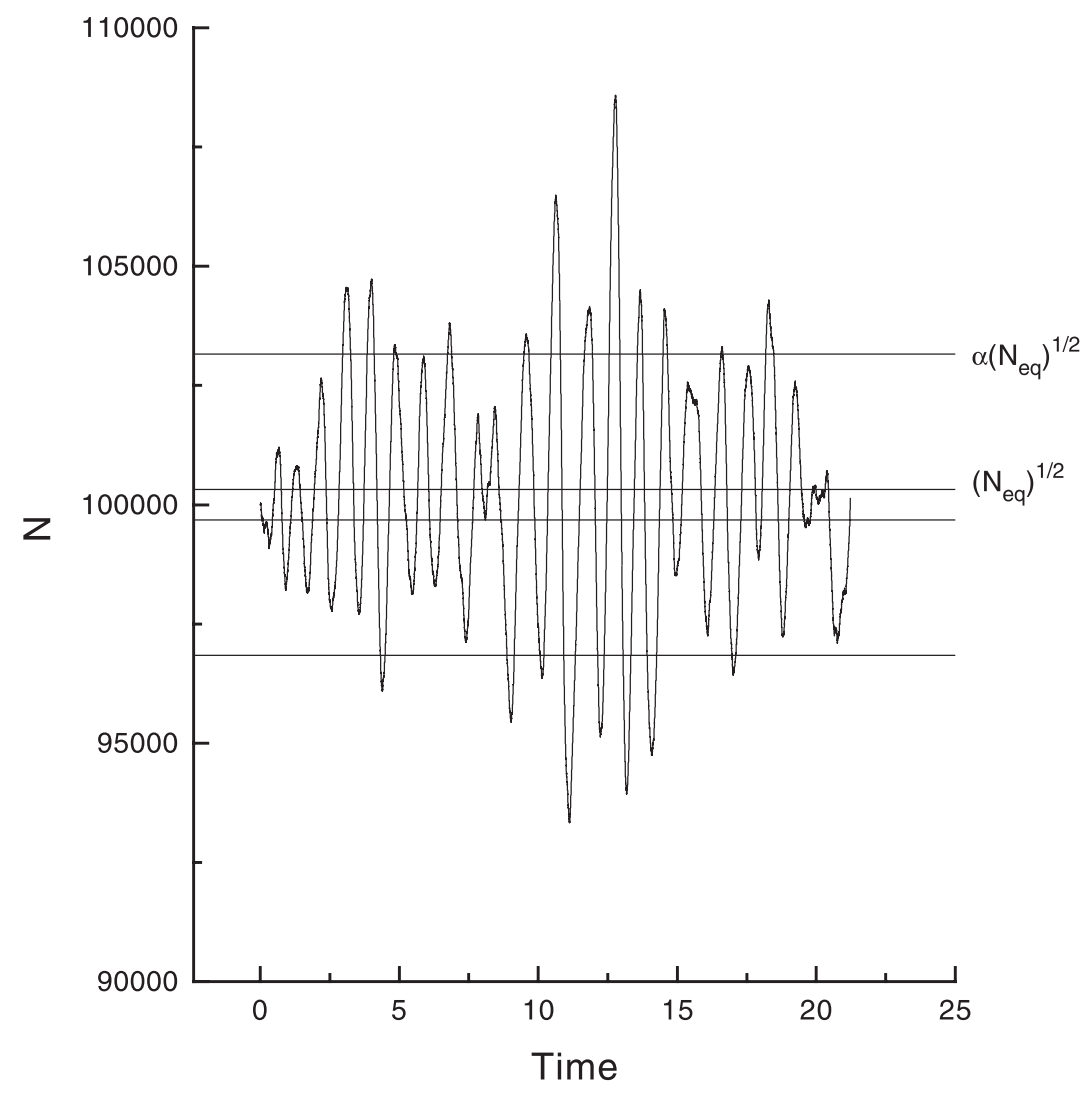

Fig. 2. Stochastic simulation. Initial conditions correspond to the deterministic equilibrium values $N_{\text {eq }}=10^{5}, n_{\text {eq }}=10^{3}$, and therefore $\alpha=10$. The horizontal lines are placed at $N_{\text {eq }} \pm \sqrt{N_{\text {eq }}}$ and $n_{\text {eq }} \pm \alpha \sqrt{N_{\text {eq }}}$. Parameter values are $a=0.1$, $b=10, \beta=10, \Omega=10^{5}$.

cannot proceed forever because far enough from the deterministic equilibrium value dominates the deterministic dynamics.

In order to obtain some quantitative results we consider the linear approximation for the (conditional) probabilities of occurrence of events

$$
\begin{aligned}
& P_{b}=\frac{1}{3}-\frac{1}{9}\left(x / N_{\mathrm{eq}}+2 y / n_{\mathrm{eq}}\right)+\mathrm{O}\left(\left(x / N_{\mathrm{eq}}\right)^{2}+\left(y / n_{\mathrm{eq}}\right)^{2}\right), \\
& P_{c}=\frac{1}{3}+\frac{1}{9}\left(2 x / N_{\mathrm{eq}}+y / n_{\mathrm{eq}}\right)+\mathrm{O}\left(\left(x / N_{\mathrm{eq}}\right)^{2}+\left(y / n_{\mathrm{eq}}\right)^{2}\right), \\
& P_{d}=\frac{1}{3}+\frac{1}{9}\left(-x / N_{\mathrm{eq}}+y / n_{\mathrm{eq}}\right)+\mathrm{O}\left(\left(x / N_{\mathrm{eq}}\right)^{2}+\left(y / n_{\mathrm{eq}}\right)^{2}\right) .
\end{aligned}
$$

A remark is pertinent at this point: the probabilities $P_{b}, P_{c}$ and $P_{d}$ are functions only of the variables $x / N_{\text {eq }}$ and $y / n_{\text {eq }}$; hence the quality of the approximation (11) depends only on the relative size of the fluctuations with respect to the equilibrium values. The scale parameter $\Omega$ only plays a role through the equilibrium values and is not essential to the approximation. 
We shall now consider the average change of the Liapunov function $E$, Eq. (4), with each event. The three contributions $\Delta E_{b}, \Delta E_{c}$ and $\Delta E_{d}$ due to each possible event are (we indicate the corresponding changes for $E_{\Gamma}$ as well in the last column):
Birth
$\Delta E_{b}=2 x+1$
$\Delta E_{\Gamma b}=\Delta E_{b}+\Gamma 2 y$
Contagion
$\Delta E_{c}=-2 x+1+2 \alpha^{2} y+\alpha^{2}$
$\Delta E_{\Gamma c}=\Delta E_{c}+\Gamma(2 x-1)$
Death
$\Delta E_{d}=-2 \alpha^{2} y+\alpha^{2}$
$\Delta E_{\Gamma d}=\Delta E_{d}+\Gamma(1-2 x-2 y)$,

where we recall that $\alpha^{2}=N_{\text {eq }} / n_{\text {eq }}$, and $N_{\text {eq }}>(1 / 4) n_{\text {eq }}$.

The mean value of the variation of the Liapunov function $E$ is

$$
\langle\Delta E\rangle \sim \Delta E_{b} P_{b}+\Delta E_{c} P_{c}+\Delta E_{d} P_{d}=\frac{2}{3}\left(\alpha^{2}+1\right)+\frac{1}{9} \frac{y}{n_{\mathrm{eq}}}\left(2 \alpha^{2}-1\right)+\frac{1}{9} \frac{x}{N_{\mathrm{eq}}}\left(\alpha^{2}+1\right)-\frac{2}{3} \frac{x^{2}}{N_{\mathrm{eq}}} .
$$

The condition of zero average variation of the Liapunov function $E,\langle\Delta E\rangle=0$, defines a parabola in phase space

$$
y=\frac{6}{\alpha^{2}\left(2 \alpha^{2}-1\right)} x^{2}-\frac{\alpha^{2}+1}{\alpha^{2}\left(2 \alpha^{2}-1\right)} x-6 n_{\mathrm{eq}} \frac{\alpha^{2}+1}{2 \alpha^{2}-1},
$$

and the intersections of the parabola with the $x$-axis for $N_{\text {eq }}, n_{\mathrm{eq}} \gg 1$ are

$$
x_{1,2} \sim \pm \sqrt{\frac{N_{\text {eq }}}{n_{\text {eq }}}} \sqrt{N_{\text {eq }}+n_{\text {eq }}}
$$

(see Fig. 3).

The deterministic equilibrium state $(x=0, y=0)$ is in the 'interior' region of the parabola, where events tend, on average, to increase $E$. As this happens, the structure of the system favors the sustenance of an oscillatory regime (it can be verified that, under the conditions $\alpha^{2}>1 / 4$, $N_{\text {eq }} \gg x \gg 1$ and $n_{\text {eq }} \gg y \gg 1$, the stochastic variable $\theta$ also increases on average).

The population state cannot remain in the interior region forever. When one of the parabola branches is crossed, events tend, on average, to decrease $E$, and then, the population state is not expected to move too far away from the deterministic equilibrium state. In the long term, the Liapunov function must fluctuate around the value

$$
E_{\mathrm{bal}}=\frac{N_{\mathrm{eq}}}{n_{\mathrm{eq}}}\left(N_{\mathrm{eq}}+n_{\mathrm{eq}}\right)
$$

representing the balance of the deterministic drive towards the equilibrium and the random walk away from it.

The intersection of the deformed circle $E=E_{\mathrm{bal}}$ with both axes gives an estimate of the fluctuations of the populations. We have

$$
\begin{aligned}
& \Delta N \sim \sqrt{\frac{N_{\mathrm{eq}}}{n_{\mathrm{eq}}}} \sqrt{N_{\mathrm{eq}}+n_{\mathrm{eq}}}, \\
& \Delta n \sim \sqrt{N_{\mathrm{eq}}+n_{\mathrm{eq}}} .
\end{aligned}
$$




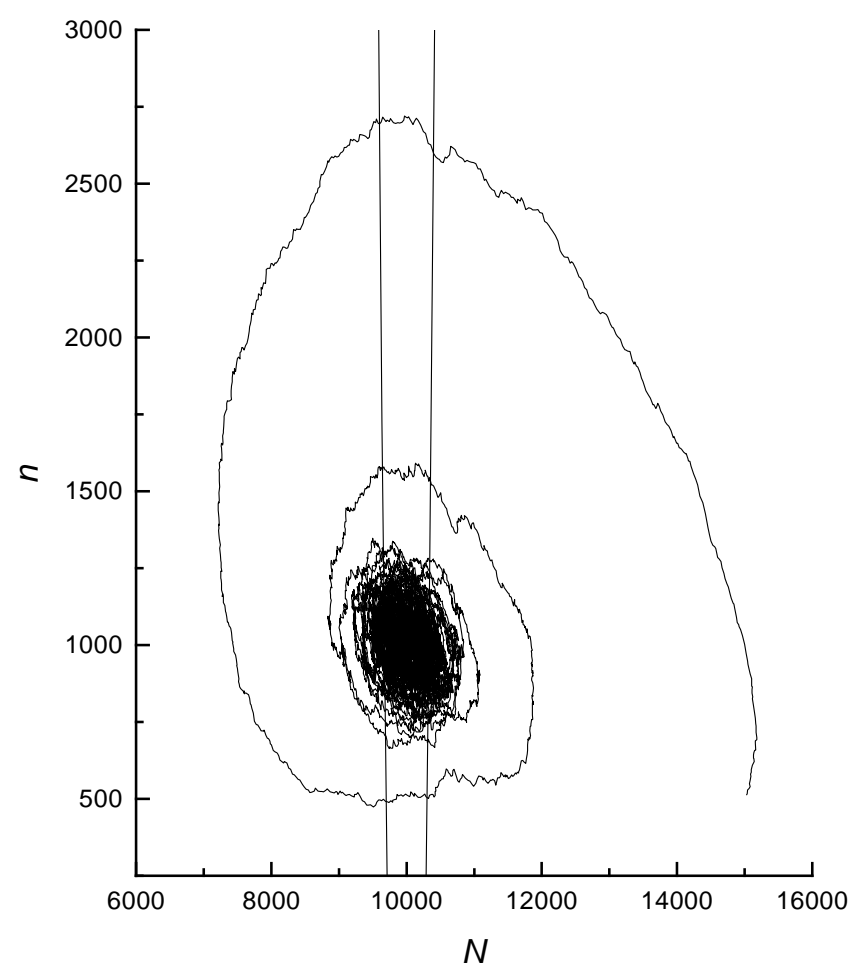

Figure 3: Phase-space plot of the stochastic simulation of Fig 1 . The almost vertical lines are actually (part of) the parabola defined by (12). Parameter values are $a=1, b=10, \beta=10, \Omega=10^{4}$.

The present estimations for the fluctuations are exactly the expressions found by Nåsell [2] for the dispersions from the mean values (here called the equilibrium values) of the marginal distribution obtained in the quasi-stationary distribution approximation.

Finally, the computation performed is consistent only when the fluctuations relative to the equilibrium values are small, i.e., we are working under the consistency conditions:

$$
\frac{\Delta N}{N_{e q}} \sim \sqrt{\frac{1}{N_{e q}}+\frac{1}{n_{e q}}} \ll 1
$$

and

$$
\frac{\Delta n}{n_{e q}} \sim \sqrt{\frac{N_{e q}}{n_{e q}^{2}}+\frac{1}{n_{e q}}} \ll 1 .
$$

Note that the condition (15) is not very restrictive, while the second one (16) imposes some requirements on the relative values of equilibrium populations. We also note that both conditions are always satisfied when the scale factor $\Omega$ is sufficiently large. 
When the Liapunov functions $E_{\Gamma}$ of the deterministic dynamics are considered, similar expressions are obtained. However, the Liapunov function increases on average in a compact region. For example, considering $E_{1 / 2}$, the average change under the transition rates (11) reads

$$
\Delta E_{1 / 2}=-\frac{1}{3 n_{\mathrm{eq}}}\left(y^{2}-\left(2 \alpha^{2}-1\right) \frac{y}{3}\right)-\frac{1}{3 N_{\mathrm{eq}}}\left(x^{2}-\left(2 \alpha^{2}-1\right) \frac{x}{6}\right)+\frac{2}{3}\left(\alpha^{2}+1\right),
$$

and the condition of zero average variation of the Liapunov function $E_{1 / 2},\left\langle\Delta E_{1 / 2}\right\rangle=0$, defines a deformed circle not centered at the equilibrium point.

Estimates of the mean amplitude of the oscillations, based on $E_{\Gamma}$, are of the same order as those obtained before (expressions (13) and (14)).

Hence, our heuristic argument implies that the deterministic equilibrium point is 'stochastically unstable', meaning that the probability of a stochastic trajectory leaving the vicinity of the equilibrium point is one provided the vicinity is in the interior of the region where $E<E_{\text {bal }}$. Furthermore, in view of the available results on stochastic stability [14-16], we conjecture that a generalization of Liapunov's instability theorem is possible, formalizing and sharpening the heuristic discussion presented here.

\section{Summary}

We have considered a two-dimensional deterministic system with an equilibrium point which is a global attractor for all the trajectories in phase space except for the invariant set $n=0$. The solutions of this system present damped oscillations towards equilibrium values. The deterministic trajectories, solutions of the deterministic model, spiral toward the equilibrium point when $N_{\text {eq }} / n_{\text {eq }}>1 / 4$.

Considering that the evolution of the system is well captured by a stochastic jump process, we observe that all the events have almost the same probability of occurrence close to the deterministic equilibrium point. Hence, the population state performs a random walk, moving away from the deterministic equilibrium point.

The Liapunov function, $E$, of the deterministic system increases (rather than decreases) on average close enough to the equilibrium point under the stochastic dynamics. The region where $E$ increases in average consists of the interior of a parabola determined by the condition $\langle\Delta E\rangle=0$.

While the population state, $(N, n)$, lies in the interior of the parabola, events tend, on average, to increase $E$. The expected oscillation amplitudes correspond to the largest (deformed) circle $E=E_{\text {bal }}$ inscribed in the parabola and its intersections with the $N$ - and $n$-axes, i.e., $\sqrt{N_{\text {eq }} / n_{\text {eq }}} \sqrt{N_{\text {eq }}+n_{\text {eq }}}$ for the $N$-population and $\sqrt{N_{\text {eq }}+n_{\text {eq }}}$ for the $n$-population give the expected fluctuations for the populations. Close to the deterministic equilibrium the stochastic behavior dominates, producing undamped oscillations.

\section{Concluding remarks}

The description of a stochastic process by a master equation for the probability distribution is a complex task when more than one population is involved. Furthermore, this picture may obscure 
some features as the persistence of oscillations. Often, analytical results are obtained by means of the so-called diffusion approximation which is valid for sufficiently large $\Omega$. We have favored here an approach closer to the jump process, which is intuitive and clearly shows the mechanisms behind the sustained oscillations. The agreement between the predicted and observed mean amplitude is very good.

We found that the deterministic equilibrium population state $\left(N_{\text {eq }}, n_{\text {eq }}\right)$, which is a global attractor for all the phase-space trajectories of the deterministic system except the invariant axis $n=0$, becomes 'stochastically unstable'.

However, for large population numbers, and far enough from the equilibrium, the deterministic dynamics drives the motion along the deterministic trajectories. Therefore, there are two regions in phase space with substantially different dynamics. One of them is pictured as the interior of the parabola where $\langle\Delta E\rangle \geqslant 0$; the other is the exterior region where the deterministic element of the dynamics dominates. By the nature of the processes, the boundary between these regions is diffuse.

The relative size of the fluctuations scales with the square root of the scale parameter $\Omega$. However, in the present approach, this parameter plays only a secondary role; the large numbers are indeed the population values at equilibrium.

We finally notice that a large $\alpha^{2}$ value implies a large ratio between the characteristic time of decay and the characteristic time of oscillations $\alpha^{2}=\omega_{0}^{2} / \gamma^{2}$. When $\alpha$ is large (slow decay) the fluctuations of the $n$ population are enhanced since the factor $\sqrt{\alpha^{2}+1}$ multiplies the standard $1 / \sqrt{n_{\mathrm{eq}}}$ term.

Finally we would like to bring to notice the fact that, since the pioneering works of Lotka and Volterra, the problem of oscillating populations has been an essential contribution to the development of modern ecological theory. Although it has been a long time since then, deterministic models continue constituting the core of most ecological thought. It is then not surprising that the oscillations of natural populations are usually explained by deterministic models whose solutions present sustained oscillations or models incorporating seasonality (the discussion by Renshaw [1, p. 204] is pertinent at this point). In the present work, we show that significant (i.e., observable and measurable) oscillations may take place even when the related deterministic dynamics show damped oscillations towards an asymptotic steady state, and these oscillations are traced to the stochastic nature of the problem.

\section{Acknowledgements}

It is a pleasure to acknowledge valuable discussions with B. Gabriel Mindlin, Mario A. Natiello and Ingemar Nåsell. We acknowledge support from the Universidad de Buenos Aires, grant TW04. J.P.A. acknowledges support from the Mathematical and Theoretical Biology Institute at Cornell University.

\section{References}

[1] E. Renshaw, Modelling Biological Populations in Space and Time, Cambridge University, Cambridge, 1991.

[2] I. Nåsell, On the time to extinction in recurrent epidemics, J. Roy. Statist. Soc. B 61 (1999) 309. 
[3] J.D. Murray, Mathematical Biology, Springer, Heidelberg, 1989.

[4] A.E. Siegman, Lasers, University Science Books, Mill Valley, 1986.

[5] H.E. Soper, Interpretation of periodicity in disease prevalence, J. Roy. Statist. Soc. A 92 (1929) 34.

[6] M.S. Bartlett, Measles periodicity and community size, J. Roy. Statist. Soc. A 120 (1957) 48.

[7] M.S. Bartlett, The critical community size for measles in the United States, J. Roy. Statist. Soc. A 123 (1960) 37.

[8] B.T. Grenfell, B. Bolker, A. Kleczkowski, Seasonality, demography and the dynamics of measles in developed countries, in: D. Mollison (Ed.), Epidemic Models: Their Structure and Relation to Data, Cambridge University, Cambridge, 1995, p. 248.

[9] M.J. Keeling, B.T. Grenfell, Disease extinction and community size: modeling the persistence of measles, Science 275 (1997) 65.

[10] O.A. van Herwaarden, J. Grasman, Stochastic epidemics: major outbreaks and the duration of the endemic period, J. Math. Biol. 33 (1995) 581.

[11] N.G. van Kampen, Stochastic Processes in Physics and Chemistry, North-Holland, Amsterdam, 1981.

[12] H.G. Solari, M.A. Natiello, B.G. Mindlin, Non-linear Dynamics: A Two-way Trip from Physics to Math, Institute of Physics, Bristol, 1996.

[13] J. Guckenheimer, P.J. Holmes, Non-linear Oscillators, Dynamical Systems and Bifurcations of Vector Fields, Springer, New York, 1986 (first printing: 1983).

[14] H.J. Kushner, Stochastic Optimization and Control, Wiley, New York, 1967, pp. 47-57 (Chapter: The concept of invariant set for stochastic dynamical systems and applications to stochastic stability).

[15] H.J. Kushner, Stability of Stochastic Dynamical Systems, in: Lecture Notes in Mathematics, vol. 294, Springer, Berlin, 1968, pp. 97-124 (Chapter: Stochastic stability).

[16] S.P. Meyn, R.L. Tweedie, Markov Chains and Stochastic Stability, Springer, London, 1993. 